\title{
PHILIPPE GLESSER
}

\section{Majoration de la norme des facteurs d'un polynôme}

Annales de la faculté des sciences de Toulouse $5^{e}$ série, tome 11, $\mathrm{n}^{\mathrm{o}} 1$ (1990), p. 67-74

$<$ http://www.numdam.org/item?id=AFST_1990_5_11_1_67_0>

(C) Université Paul Sabatier, 1990, tous droits réservés.

L'accès aux archives de la revue «Annales de la faculté des sciences de Toulouse » (http://picard.ups-tlse.fr/ annales/) implique l'accord avec les conditions générales d'utilisation (http://www.numdam.org/conditions). Toute utilisation commerciale ou impression systématique est constitutive d'une infraction pénale. Toute copie ou impression de ce fichier doit contenir la présente mention de copyright.

\section{NumDam}

Article numérisé dans le cadre du programme

Numérisation de documents anciens mathématiques

http://www.numdam.org/ 


\section{Majoration de la norme des facteurs d'un polynôme}

PhILIPPE Glesser ${ }^{(1)}$

REsume. - Le but de cet article est de donner une nouvelle majoration de la norme des facteurs d'un polynôme à coefficients complexes. Outre son intérêt théorique, une telle majoration est utile pour les algorithmes de factorisation des polynômes.

Abstract. - The object of this paper is to give a new upper bound for the norm of factors of a polynomial with complex coefficients. Besides its theoretical interest, this kind of bound is useful in algorithms for polynomial factorisation.

\section{Introduction}

Dans cet article on utilise les définitions suivantes :

Soit $F$ un polynôme à coefficients complexes, $F=\sum_{i=0}^{n} a_{i} X^{i}$, on note :

$$
\begin{aligned}
H(F) & =\max _{i=0,1, \ldots, n}\left|a_{i}\right|, \text { la hauteur de } F, \\
L(F) & =\sum_{i=0}^{n}\left|a_{i}\right|, \text { la longueur de } F, \\
\text { et }\|F\|_{2} & =\left(\sum_{i=0}^{n}\left|a_{i}\right|^{2}\right)^{\frac{1}{2}} \text {, la norme de } F .
\end{aligned}
$$

Dans la suite on considère deux polynômes $P$ et $Q$ à coefficients complexes,

$$
\begin{aligned}
& P=a_{m} X^{m}+a_{m-1} X^{m-1}+\cdots+a_{0}, \\
& Q=b_{n} X^{n}+b_{n-1} X^{n-1}+\cdots+b_{0},
\end{aligned}
$$

$P$ étant un diviseur de $Q$.

(1) Ph. Glesser, Université Louis-Pasteur, 7, rue René-Descartes 67084 Strasbourg 
Le but de cet article est de majorer la longueur, la norme et la hauteur de $P$, en fonction de la norme de $Q$. On utilisera pour cela un lemme dû à A. Granville.

\section{Résultats intermédiaires}

Cette partie comprend l'énoncé et la démonstration de deux lemmes et de deux corollaires nécessaires à la démonstration du résultat principal de cet article qui se trouve, sous forme de théorème, dans la troisième partie du papier.

LEMME 1. - Soit $P$ un polynôme à coefficients dans C :

$$
P(X)=a_{0}+a_{1} X+\cdots+a_{m} X^{m} .
$$

On pose : $\quad(X+\alpha) P(X)=b_{0}+b_{1} X+\cdots+b_{m+1} X^{m+1}$, et $\quad(\bar{\alpha} X+1) P(X)=c_{0}+c_{1} X+\cdots+c_{m+1} X^{m+1}$.

Alors : $\quad\|(X+\alpha) P(X)\|_{2}=\|(\bar{\alpha} X+1) P(X)\|_{2}$,

tí de plus si $|\alpha| \leq 1$, alors :

$$
\sum_{i=0}^{k}\left|c_{i}\right|^{2} \geq \sum_{i=0}^{k}\left|b_{i}\right|^{2} \quad(0 \leq k \leq m) .
$$

Preuve. - Ayant posé $a_{-1}=0$ et $a_{m+1}=0$, on a :

$$
b_{i}=a_{i-1}+\alpha a_{i} \text { et } c_{i}=\bar{\alpha} a_{i-1}+a_{i}, \quad \text { pour } 0 \leq i \leq m+1 .
$$

Donc : $\sum_{i=0}^{k}\left|c_{i}\right|^{2}=\sum_{i=0}^{k}\left(\bar{\alpha} a_{i-1}+a_{i}\right)\left(\alpha \overline{a_{i-1}}+\overline{a_{i}}\right)$

$$
=\sum_{i=0}^{k}\left(|\alpha|^{2}\left|a_{i-1}\right|^{2}+\bar{\alpha} \overline{a_{i}} a_{i-1}+\alpha a_{i} \overline{a_{i-1}}+\left|a_{i}\right|^{2}\right) .
$$

Tandis que : $\sum_{i=0}^{k}\left|b_{i}\right|^{2}=\sum_{i=0}^{k}\left(\left|a_{i-1}\right|^{2}+\alpha a_{i} \overline{a_{i-1}}+\overline{\alpha a_{i}} a_{i-1}+|\alpha|^{2}\left|a_{i}\right|^{2}\right)$. 
Ainsi :

$$
\begin{aligned}
\sum_{i=0}^{k}\left|c_{i}\right|^{2}-\sum_{i=0}^{k}\left|b_{i}\right|^{2} & =\left|a_{k}\right|^{2}-|\alpha|^{2}\left|a_{k}\right|^{2} \\
& =\left(1-|\alpha|^{2}\right)\left|a_{k}\right|^{2}
\end{aligned}
$$

Ceci démontre la première assertion en faisant $k=m+1$, et la seconde pour $0 \leq k \leq m$, lorsque $|\alpha| \leq 1$.

Le corollaire suivant est une conséquence immédiate du lemme 1.

Corollaire 1. - Soit $P$ un polynôme à coefficients dans C :

$$
P(X)=a_{m}\left(X+\alpha_{1}\right) \ldots\left(X+\alpha_{m}\right)=a_{0}+a_{1} X+\cdots+a_{m} X^{m} .
$$

On pose : $\widetilde{P}(X)=a_{m} \prod_{S}\left(\overline{\alpha_{i}} X+1\right) \prod_{\bar{S}}\left(X+\alpha_{i}\right)$, où $S$ est un sous-ensemble de $\{1,2, \ldots, m\}$ tel que si $i \in S,\left|\alpha_{i}\right| \leq 1, \bar{S}$ étant son complémentaire dans $\{1,2, \ldots, m\}$.

Alors : $\quad\|P\|_{2}=\|\widetilde{P}\|_{2}$ et $\sum_{i=0}^{k}\left|\widetilde{a}_{i}\right|^{2} \geq \sum_{i=0}^{k}\left|a_{i}\right|^{2}, \quad(0 \leq k \leq m)$

(les $\tilde{a}_{i}$ sont les coefficients de $\widetilde{P}$ ).

Lemme 2 (A. Granville). - Soit $P$ et $Q$ deux polynômes à coeffcients complexes :

$$
\begin{aligned}
& P=a_{m}\left(X+\alpha_{1}\right) \ldots\left(X+\alpha_{m}\right)=a_{m} X^{m}+\cdots+a_{0} . \\
& Q=b_{n}\left(X+\alpha_{1}\right) \ldots\left(X+\alpha_{n}\right)=b_{n} X^{n}+\cdots+b_{0}, \quad n>m, b_{n} \neq 0
\end{aligned}
$$

(autrement dit $P$ divise $Q$ ).

On suppose que $\left|\alpha_{i}\right| \geq 1(m+1 \leq i \leq n)$. Alors :

$$
\left|a_{j}\right| \leq \sum_{i=0}^{j}\left|b_{i}\right|\left(\begin{array}{c}
j-i+n-m-1 \\
j-i
\end{array}\right)\left|\frac{a_{0}}{b_{0}}\right| \quad(0 \leq j \leq m) .
$$

Preuve. - On a $: P=\frac{a_{m}}{b_{n}} \frac{1}{\left(X+\alpha_{m+1}\right)} \cdots \frac{1}{\left(X+\alpha_{n}\right)} Q$. 


\section{Philippe Glesser}

Or $\frac{1}{X+\alpha_{i}}=\frac{1}{\alpha_{i}\left(X / \alpha_{i}+1\right)}=\frac{1}{\alpha_{i}}\left(1-\frac{X}{\alpha_{i}}+\left(\frac{X}{\alpha_{i}}\right)^{2}+\cdots\right)$,

ce qui entraîne que :

$$
\frac{1}{X+\alpha_{i}} \ll \frac{1}{\left|\alpha_{i}\right|}\left(1+X+X^{2}+X^{3}+\cdots\right)
$$

(si $U=\sum_{n \geq 0} u_{n} X^{n}$ est une série formelle à coefficients complexes et $V=\sum_{n \geq 0} v_{n} X^{n}$ une série formelle à coefficients réels positifs ou nuls, on dit que $V$ majore $U$ et on note $U \ll V$, si $\left|u_{n}\right| \leq v_{n}$ pour tout n positif ou nul). Donc :

$$
P \ll\left|\frac{a_{0}}{b_{0}}\right|\left(1+X+X^{2}+X^{3}+\cdots\right)^{n-m}\left(\left|b_{0}\right|+\cdots+\left|b_{n}\right| X^{n}\right),
$$

ce qui entraîne que :

$$
P \ll\left|\frac{a_{0}}{b_{0}}\right|\left(\sum_{i \geq 0}\left(\begin{array}{c}
n-m+i-1 \\
i
\end{array}\right) X^{i}\right)\left(\left|b_{0}\right|+\cdots+\left|b_{n}\right| X^{n}\right),
$$

et par conséquent que :

$$
P \ll\left|\frac{a_{0}}{b_{0}}\right| \sum_{j \geq 0}\left(\sum_{i=0}^{j}\left|b_{i}\right|\left(\begin{array}{c}
n-m+j-i-1 \\
j-i
\end{array}\right)\right) X^{j},
$$

d'où l'on déduit finalement que :

$$
\left|a_{j}\right| \leq\left|\frac{a_{0}}{b_{0}}\right| \sum_{i=0}^{j}\left|b_{i}\right|\left(\begin{array}{c}
n-m+j-i-1 \\
j-i
\end{array}\right) .
$$

Corollaire 2. - Soient $P$ et $Q$ deux polynômes à coefficients complexes:

$$
\begin{aligned}
& P=a_{m}\left(X+\alpha_{1}\right) \ldots\left(X+\alpha_{m}\right)=a_{m} X^{m}+\cdots+a_{0} \\
& Q=b_{n}\left(X+\alpha_{1}\right) \ldots\left(X+\alpha_{n}\right)=b_{n} X^{n}+\cdots+b_{0}, \quad n>m, b_{0} \neq 0 .
\end{aligned}
$$

Alors : $\quad\left|a_{j}\right| \leq\left|\frac{a_{0}}{b_{0}}\right|\left(\begin{array}{c}j+n-m \\ j\end{array}\right)\|Q\|_{2} \quad(0 \leq j \leq m)$. 
Preuve. - Soit :

$$
\widetilde{Q}=b_{n} \prod_{\substack{\left|\alpha_{i}\right| \geq 1 \\ i \leq m \\ i \leq m}}\left(X+\alpha_{i}\right) \prod_{\substack{\left|\alpha_{i}\right|<1 \\ \text { et } \\ i>m}}\left(\bar{\alpha}_{i} X+1\right)
$$

D'après le lemme 2: $\quad\left|a_{j}\right| \leq\left|\frac{a_{0}}{\widetilde{b}_{0}}\right| \sum_{i=0}^{j}\left|\tilde{b}_{i}\right|\left(\begin{array}{c}j-i+n-m-1 \\ j-i\end{array}\right)$, où les $\widetilde{b}_{i}$ sont les coefficients de $\widetilde{Q}$. On vérifie que $\left|b_{0}\right| \leq\left|\widetilde{b}_{0}\right|$, d'où l'on déduit que :

$$
\left|a_{j}\right| \leq H(\tilde{Q})\left|\frac{a_{0}}{b_{0}}\right| \sum_{i=0}^{j}\left(\begin{array}{c}
j-i+n-m-1 \\
j-i
\end{array}\right) .
$$

Donc: $\quad\left|a_{j}\right| \leq\left|\frac{a_{0}}{b_{0}}\right|\left(\begin{array}{c}j+n-m \\ j\end{array}\right)\|\widetilde{Q}\|_{2}$

et par conséquent :

$$
\left|a_{j}\right| \leq\left|\frac{a_{0}}{b_{0}}\right|\left(\begin{array}{c}
j+n-m \\
j
\end{array}\right)\|Q\|_{2},
$$

d'après le corollaire 1 .

\section{3. Énoncé et démonstration du théorème}

THÉORÈme - - Soient $P$ et $Q$ deux polynômes à coefficients complexes :

$$
\begin{aligned}
& P=a_{m}\left(X+\alpha_{1}\right) \ldots\left(X+\alpha_{m}\right)=a_{m} X^{m}+\cdots+a_{0}, \\
& Q=b_{n}\left(X+\alpha_{1}\right) \ldots\left(X+\alpha_{n}\right)=b_{n} X^{n}+\cdots+b_{0}, \quad n \geq m, b_{0} \neq 0 .
\end{aligned}
$$

Alors :

$$
\begin{aligned}
& L(P) \leq\left(\left|\frac{a_{0}}{b_{0}}\right|+\left|\frac{a_{m}}{b_{n}}\right|\right)\left(\begin{array}{c}
n-[(m-1) / 2] \\
{[m / 2]}
\end{array}\right)\|Q\|_{2}, \\
&\|P\|_{2} \leq \sqrt{\left|\frac{a_{0}}{b_{0}}\right|^{2}+\left|\frac{a_{m}}{b_{n}}\right|^{2}}\left(\sum_{j=0}^{[m / 2]}\left(\begin{array}{c}
j+n-m \\
j
\end{array}\right)^{2}\right)^{\frac{1}{2}}\|Q\|_{2}, \\
& \text { et } \quad H(P) \leq \max \left\{\left|\frac{a_{0}}{b_{0}}\right|,\left|\frac{a_{m}}{b_{n}}\right|\right\}\left(\begin{array}{c}
n-[m+1) / 2] \\
{[m / 2]}
\end{array}\right)\|Q\|_{2} . \\
&-71-
\end{aligned}
$$


Preuve. - D'après le corollaire 2 :

$$
\left|a_{j}\right| \leq\left|\frac{a_{0}}{b_{0}}\right|\left(\begin{array}{c}
j+n-m \\
j
\end{array}\right)\|Q\|_{2}, \quad \text { pour } 0 \leq j \leq[m / 2] .
$$

En regardant les polynômes réciproques de $P$ et $Q$, c'est-à-dire respectivement $a_{0} X^{m}+\cdots+a_{m}$ et $b_{0} X^{n}+\cdots+b_{n}$, on constate de même que :

$$
\left|a_{m-j}\right| \leq\left|\frac{a_{m}}{b_{n}}\right|\left(\begin{array}{c}
j+n-m \\
j
\end{array}\right)\|Q\|_{2}, \quad \text { pour } 0 \leq j \leq[m / 2] .
$$

Donc : $L(P)=\sum_{j=0}^{m}\left|a_{j}\right| \leq\left(\left|\frac{a_{0}}{b_{0}}\right|+\left|\frac{a_{m}}{b_{n}}\right|\right) \sum_{j=0}^{[m / 2]}\left(\begin{array}{c}j+n-m \\ j\end{array}\right)\|Q\|_{2}$, d'où l'on déduit : $L(P) \leq\left(\left|\frac{a_{0}}{b_{0}}\right|+\left|\frac{a_{m}}{b_{n}}\right|\right)\left(\begin{array}{c}n-m+[m / 2]+1 \\ {[m / 2]}\end{array}\right)\|Q\|_{2}$.

Les deux autres inégalités se démontrent de la même manière.

Ce théorème a, pour le cas particulièrement intéressant des polynômes à coefficients entiers, la conséquence immédiate suivante.

Corollaire 3. - Soient $P$ et $Q$ deux polynômes à coefficients entiers tels que $P$ divise $Q$ ( $m=$ degré de $P$ et $n=$ degré de $Q, n \geq m)$, alors :

$$
\begin{aligned}
L(P) & \leq 2\left(\begin{array}{c}
n-[(m-1) / 2] \\
{[m / 2]}
\end{array}\right)\|Q\|_{2}, \\
\|P\|_{2} & \leq\left(2 \sum_{j=0}^{[m / 2]}\left(\begin{array}{c}
j+n-m \\
j
\end{array}\right)\right)^{2}\|Q\|_{2}, \\
\text { et } \quad H(P) & \leq\left(\begin{array}{c}
n-[(m+1) / 2] \\
{[m / 2]}
\end{array}\right)\|Q\|_{2} .
\end{aligned}
$$

Corollaire 4. - Soient $P$ et $Q$ deux polynômes à coefficients complexes :

$$
\begin{aligned}
& P=a_{m} X^{m}+\cdots+a_{0}, \\
& Q=b_{n} X^{n}+\cdots+b_{0} .
\end{aligned}
$$

On suppose que $P$ divise $Q$ et que $\left|a_{0}\right| \leq\left|b_{0}\right|$ et $\left|a_{m}\right| \leq\left|b_{n}\right|$, alors :

$$
L(P) \leq 2^{2 n / 3}\|Q\|_{2}, \quad \text { lorsque } n \text { est assez grand. }
$$


Preuve. - 1) On suppose que $m \geq 2 n / 3$.

D'après le théorème : $L(P) \leq 2\left(\begin{array}{c}n-[(m-1) / 2] \\ {[m / 2]}\end{array}\right)\|Q\|_{2}$.

Or comme $m \geq \frac{2 n}{3}: \quad L(P) \leq 2\left(\begin{array}{c}2 n / 3+2 \\ n / 3+1\end{array}\right)\|Q\|_{2}$.

Enfin : $2\left(\begin{array}{c}2 n / 3+2 \\ n / 3+1\end{array}\right) \sim \frac{8}{\sqrt{\pi(n / 3+1)}} 2^{2 n / 3}$, lorsque $n$ tend vers l'infini.

2) On suppose que $m \leq 2 n / 3:$ La majoration classique $L(P) \leq 2^{m}\|Q\|_{2}$ montre que :

$$
L(P) \leq 2^{2 n / 3}\|Q\|_{2} .
$$

\section{Conclusion}

L'intérêt du théorème est de fournir une bonne majoration de la taille $\mathrm{du}$ facteur $P$ de degré $m$ d'un polynôme $Q$ de degré $m+k$, lorsque le rapport du degré de $P$ par le degré de $Q$ est proche de 1.

Une telle majoration peut être utile pour comparer la mesure et la hauteur d'un polynôme donné (cf. [3] et [6]). La majoration utilisée dans [3] était la suivante :

$$
H(P) \leq \frac{(m+k)^{m+k}}{m^{m} k^{k}} L(Q)
$$

(remarque : d'autres inégalités de ce type sont données dans [1], [2] et [5]).

Comparons-la avec la majoration induite par le théorème dans le cas où $\left|a_{0}\right| \leq\left|b_{0}\right|$ et $\left|a_{m}\right| \leq\left|b_{m+k}\right|$ :

$$
H(P) \leq\left(\begin{array}{c}
{[m / 2]+k} \\
{[m / 2]}
\end{array}\right) L(Q) .
$$

On suppose que $k=\mathrm{O}\left(\mathrm{m}^{2 / 3}\right)$ en l'infini. On montre que :

$$
\frac{(m+k)^{m+k}}{m^{m} k^{k}} \sim\left(\frac{e m}{k}\right)^{k} e^{\frac{k^{2}}{2 m}}
$$

alors que :

$$
\left(\begin{array}{c}
{[m / 2]+k} \\
{[m / 2]}
\end{array}\right) \sim \frac{1}{\sqrt{2 \pi k}}\left(\frac{e}{k}\left[\frac{m}{2}\right]\right)^{k} e^{\frac{k^{2}}{2[m / 2]}} \quad \text { lorsque } m \text { tend vers l'infini. }
$$

Donc, lorsque $m$ est assez grand, l'inégalité (2) est meilleure que l'inégalité (1). 


\section{Exemples numériques}

En testant différentes valeurs de $m$ et de $k$, on obtient les tableaux suivants :

\begin{tabular}{|l|c|c|c|c|c|}
\hline$@ 1$ & 10 & 20 & 30 & 50 & 100 \\
\hline 1 & 25,8 & 53,0 & 80,2 & 134,5 & 270,5 \\
\hline 3 & 449,7 & 4695 & 17197 & 84827 & 710850 \\
\hline 6 & & 202255 & $3,31 \cdot 10^{6}$ & $9,28 \cdot 10^{7}$ & $7,19 \cdot 10^{9}$ \\
\hline 10 & & & $1,96 \cdot 10^{8}$ & $7,35 \cdot 10^{10}$ & $1,31 \cdot 10^{14}$ \\
\hline
\end{tabular}

\begin{tabular}{|l|r|r|r|c|c|}
\hline$@ 1$ & 10 & \multicolumn{1}{|c|}{20} & 30 & 50 & 100 \\
\hline 1 & 5 & 10 & 15 & 25 & 50 \\
\hline 3 & 20 & 165 & 560 & 2600 & 20825 \\
\hline 6 & & 1716 & 18564 & 376740 & $2,296 \cdot 10^{7}$ \\
\hline 10 & & & 184756 & $3,005 \cdot 10^{7}$ & $2,29 \cdot 10^{10}$ \\
\hline
\end{tabular}

Le premier tableau donne les valeurs de : $\frac{(m+k)^{m+k}}{m^{m} k^{k}}$, le second donne les valeurs de : $\left(\begin{array}{c}{[m / 2]+k} \\ {[m / 2]}\end{array}\right)$.

\section{Remerciements.}

Je remercie M. Mignotte pour ses nombreuses remarques.

\section{Références}

[1] J.D. DONALDSON and Q.I. RAHMAN .- Inequalities for polynomials whith a prescribed zero, Pacific J. Math. 41 (1983) pp. 375-378

[2] A. Durand .- A Propos d'un théorème de S. Bernstein sur la dérivée d'un polynôme, C.R. Acad. Sci. Paris Sér. I Math. 290 (1980) pp. 523-525

[3] PH. Glesser . - Inégalités sur la mesure des polynômes, Rendiconti-Cagliari (à paraître)

[4] A. Granville Ph. D thesis (manuscrit)

[5] R. GÜ TING .- Polynomials whith multiple zeroes, Mathematika 14 (1967) pp. 181-196

[6] M. MignotTe . - An inequality about irreducible factors of integer polynomials, Journal of number theory (1988) pp. 156-166 